PROCEEDINGS OF THE

AMERICAN MATHEMATICAL SOCIETY

Volume 125, Number 9, September 1997, Pages 2703-2709

S 0002-9939(97)03995-6

\title{
COLLAPSING SUCCESSORS OF SINGULARS
}

\author{
JAMES CUMMINGS
}

(Communicated by Andreas R. Blass)

\begin{abstract}
Let $\kappa$ be a singular cardinal in $V$, and let $W \supseteq V$ be a model such that $\kappa_{V}^{+}=\lambda_{W}^{+}$for some $W$-cardinal $\lambda$ with $W \models \operatorname{cf}(\kappa) \stackrel{2 f}{ }(\lambda)$. We apply Shelah's pcf theory to study this situation, and prove the following results.

1) $W$ is not a $\kappa^{+}$-c.c generic extension of $V$.

2) There is no "good scale for $\kappa$ " in $V$, so in particular weak forms of square must fail at $\kappa$.

3) If $V \models \operatorname{cf}(\kappa)=\aleph_{0}$ then $V \models " \kappa$ is strong limit $\Longrightarrow 2^{\kappa}=\kappa^{+"}$, and also

$\omega_{\kappa} \cap W \supsetneq{ }^{\omega} \kappa \cap V$.

4) If $\kappa=\aleph_{\omega}^{V}$ then $\lambda \leq\left(2^{\aleph_{0}}\right)_{V}$.
\end{abstract}

\section{InTRODUCTION}

The results in this paper were motivated by the following natural question.

Question 1. Can there exist a pair of inner models of ZFC, $V$ and $W$ say, such that $V \subseteq W$ and $\aleph_{\omega+1}^{V}=\aleph_{2}^{W}$ ?

This question was raised by Bukovský and Copláková-Hartová [1] who observed (Theorem 1.7 of [1]) that if we have $V$ and $W$ as above then $W \models 2^{\aleph_{0}} \geq \aleph_{2}$, because $V \models \aleph_{\omega}^{\aleph_{0}} \geq \aleph_{\omega+1}$. They also note that if Question 1 has a positive answer then it is possible to violate $\mathrm{CH}$ by adding a real.

We can ask more general questions about the possibilities for collapsing successors of singulars; for example

Question 2. Let $V \models$ " $\kappa$ is singular". Can there be $W \supseteq V$ such that

$$
W \models " \kappa_{V}^{+} \text {is a cardinal and } \operatorname{cf}(\kappa) \neq \operatorname{cf}(|\kappa|) " ?
$$

The restriction " $\operatorname{cf}(\kappa) \neq \operatorname{cf}(|\kappa|)$ " is not unreasonable, because for example it is easy to collapse $\aleph_{\omega .2+1}^{V}$ to $\aleph_{\omega+1}^{V[G]}$ by forcing with $\operatorname{Coll}\left(\omega, \aleph_{\omega}\right)$.

Work of Shelah [10] shows that a positive solution to Question 1 or Question 2 would require the use of large cardinals.

Definition 1.1. Let $\kappa$ be a cardinal. Then $\mathrm{AD}_{\kappa}$ holds iff there exists a sequence $\left\langle A_{\alpha}: \alpha<\kappa^{+}\right\rangle$such that

1. For each $\alpha<\kappa^{+}, A_{\alpha}$ is an unbounded subset of $\kappa$.

2. For each $\beta<\kappa^{+}$there exists $g: \beta \longrightarrow \kappa$ such that $\left\langle A_{\alpha} \backslash g(\alpha): \alpha<\beta\right\rangle$ is a sequence of mutually disjoint sets.

Received by the editors March 20, 1996.

1991 Mathematics Subject Classification. Primary 03E05; Secondary 03E35.

Key words and phrases. Squares, pcf, successors of singulars, good points.

(c)1997 American Mathematical Society 
Shelah proved ([10], page 440) that

1. If $\kappa$ is regular, or if $\kappa$ is singular and $\square_{\kappa}$ holds, then $\mathrm{AD}_{\kappa}$ holds.

2. If $V \models \mathrm{AD}_{\kappa}, W \supseteq V$ and $W \models$ " $\kappa_{V}^{+}$is a cardinal", then $W=\operatorname{cf}(\kappa)=\operatorname{cf}(|\kappa|)$. In particular, if $V \models \mathrm{AD}_{\kappa}+\operatorname{cf}(\kappa)=\omega$ then $W \models \operatorname{cf}(|\kappa|)=\omega$, so that in $W$ the cardinal $\kappa_{V}^{+}$must be $\aleph_{1}^{W}$ or the successor of a cardinal of cofinality $\omega$.

Remark 1.2. For $\kappa$ singular the weak square principle $\square_{\kappa}^{*}$ is enough to derive $\mathrm{AD}_{\kappa}$. See [3] for a proof.

The connection with large cardinals arises because for $\kappa$ singular the principle $\square_{\kappa}^{*}$ holds unless there exist inner models of some very strong large cardinal axioms (at the level of Woodin cardinals). See [6], [7] and [8].

The results in this paper are more general than Shelah's in the sense that they can be applied in some situations where $V \models \neg \mathrm{AD}_{\kappa}$. See [4] and [3] for a discussion of the implications between $\mathrm{AD}_{\kappa}$, squares, scales and reflection principles.

The author would like to thank Matt Foreman and Menachem Magidor for many interesting and informative conversations about the connections between squares and pcf theory. Thanks also to Doug Burke for his incisive comments on the first version of this paper.

\section{PCF TheORY, GOOD POINTS AND APPROACHABILITY}

In this section we review some facts about pcf theory that will be used later. For a detailed development of pcf theory see [2] or [11]. All the facts quoted here are due to Shelah unless otherwise stated.

Definition 2.1. Let $\kappa$ be a singular cardinal. A scale for $\kappa$ (of length $\kappa^{+}$) is $(\vec{\kappa}, \vec{f})$ where

1. $\vec{\kappa}=\left\langle\kappa_{i}: i<\operatorname{cf}(\kappa)\right\rangle$ is a strictly increasing sequence of regular cardinals with $\sup _{i} \kappa_{i}=\kappa$.

2. $\vec{f}=\left\langle f_{\alpha}: \alpha<\kappa^{+}\right\rangle$is a sequence such that

(a) $f_{\alpha} \in \prod_{i} \kappa_{i}$.

(b) $\vec{f}$ is strictly increasing and cofinal in $\left(\prod_{i} \kappa_{i},<^{*}\right)$ where $<^{*}$ is the eventual domination relation on $\prod \kappa_{i}$.

Fact 2.2. If $\kappa$ is singular then there exists a scale of length $\kappa^{+}$for $\kappa$.

Definition 2.3. Let $(\vec{\kappa}, \vec{f})$ be a scale of length $\kappa^{+}$for $\kappa . \alpha<\kappa^{+}$is good (for $\left.\vec{f}\right)$ iff there exist $A \subseteq \alpha$ unbounded in $\alpha$ and $i<\operatorname{cf}(\kappa)$ such that whenever $\beta, \gamma \in A$ with $\beta<\gamma$ and $j>i$ then $f_{\beta}(j)<f_{\gamma}(j)$.

Remark 2.4. If $\operatorname{cf}(\alpha)<\operatorname{cf}(\kappa)$ then it is easy to see that $\alpha$ is good.

Remark 2.5. If $\operatorname{cf}(\alpha)>\operatorname{cf}(\kappa), \alpha$ being good is equivalent to $\left\langle f_{\beta}: \beta<\alpha\right\rangle$ having a least upper bound $g$ such that $\operatorname{cf}(g(i))=\operatorname{cf}(\alpha)$ for all large $i$.

Remark 2.6. The definition of good point actually makes sense for any $\vec{\kappa}$ and any increasing sequence from $\left(\prod_{i} \kappa_{i},<^{*}\right)$.

Fact 2.7. If $\rho=\operatorname{cf}(\rho)<\kappa$ and $(\vec{\kappa}, \vec{f})$ is a scale of length $\kappa^{+}$for $\kappa$ then

$$
\left\{\alpha<\kappa^{+}: \operatorname{cf}(\alpha)=\rho \text { and } \alpha \text { is good }\right\}
$$

is stationary in $\kappa^{+}$. 
Since we will use some of the ideas later, we sketch a proof of this fact.

Proof. We prove it first when $\operatorname{cf}(\kappa)<\rho$. Let $C \subseteq \kappa^{+}$be club. Fix $\theta$ some large regular cardinal. Build an increasing and continuous sequence $\left\langle N_{i}: i<\rho\right\rangle$ such that

1. $N_{i} \prec\left(H_{\theta}, \in,<_{\theta}\right)$ where $<_{\theta}$ is some wellordering of $H_{\theta}$.

2. $C, \vec{\kappa}, \vec{f} \in N_{0}$ and $\operatorname{cf}(\kappa) \subseteq N_{0}$.

3. $\left|N_{i}\right|<\rho$.

4. $\left\langle N_{i}: i \leq j\right\rangle \in N_{j+1}$ for all $j<\rho$ (it will follow that $i \subseteq N_{i}$ and hence that $i<j \Longrightarrow N_{i} \in N_{j}$ ).

Notice that if $i<j$ then $\sup \left(N_{i} \cap \kappa^{+}\right) \in N_{j}$, so that $\left\langle\sup \left(N_{i} \cap \kappa^{+}\right): i<\rho\right\rangle$ is increasing and continuous. The limit of this sequence is $\sup \left(\bigcup_{i} N_{i} \cap \kappa^{+}\right)$. It follows from elementarity that $C$ is unbounded in $N_{i} \cap \kappa^{+}$, so that $\sup \left(N_{i} \cap \kappa^{+}\right) \in C$.

Define $\chi_{i} \in \prod_{n} \kappa_{n}$ by setting $\chi_{i}(n)=0$ for $\kappa_{n}<\rho, \chi_{i}(n)=\sup \left(N_{i} \cap \kappa_{n}\right)$ for $\kappa_{n} \geq \rho$. Since $N_{i} \in N_{i+1}$ we see that $\chi_{i} \in N_{i+1}$, so that there exists $\alpha_{i} \in N_{i+1} \cap \kappa^{+}$ such that $\alpha_{i}>\sup \left(N_{i} \cap \kappa^{+}\right)$and $\chi_{i}<^{*} f_{\alpha_{i}}$. Since $\operatorname{cf}(\kappa) \subseteq N_{i+1}$ we see that for all $n<\operatorname{cf}(\kappa)$ we have $f_{\alpha_{i}}\left(\kappa_{n}\right) \in N_{i+1} \cap \kappa_{n}$, in particular we have $f_{\alpha_{i}}<^{*} \chi_{i+1}$.

Now for every $i<\rho$ we may choose $j<\operatorname{cf}(\kappa)$ such that

$$
k>j \Longrightarrow \chi_{i}(k)<f_{\alpha_{i}}(k)<\chi_{i+1}(k) \text {. }
$$

Since $\rho>\operatorname{cf}(\kappa)$ we may fix $A \subseteq \rho$ unbounded and $j<\operatorname{cf}(\kappa)$ such that

$$
\forall i \in A \forall k>j \chi_{i}(k)<f_{\alpha_{i}}(k)<\chi_{i+1}(k) .
$$

It follows that if $i_{0}, i_{1} \in A$ with $i_{0}<i_{1}$ then for $k>j$ we have

$$
f_{\alpha_{i_{0}}}(k)<\chi_{i_{0}+1}(k) \leq \chi_{i_{1}}(k)<f_{\alpha_{i_{1}}}(k) .
$$

Now we know that $\sup \left(N_{i} \cap \kappa^{+}\right)<\alpha_{i}<\sup \left(N_{i+1} \cap \kappa^{+}\right)$, so this shows that $\sup _{i} \alpha_{i}=\sup _{i} \sup \left(N_{i} \cap \kappa^{+}\right)$is a good point. This point is also a point of $C$ with cofinality $\rho$, so we have shown the set of good points of cofinality $\rho$ to be stationary. This concludes the proof when $\rho>\operatorname{cf}(\kappa)$.

To finish we observe that if $A$ witnesses $\alpha$ to be a good point for $\vec{f}$, and $\beta$ is a limit point of $A$, then $A \cap \beta$ witnesses that $\beta$ is good for $\vec{f}$. Given $\rho=\operatorname{cf}(\rho) \leq \operatorname{cf}(\kappa)$ and a club set $C$, we first use the argument above to find $\alpha \in \lim (C)$ with $\operatorname{cf}(\alpha)=\operatorname{cf}(\kappa)^{+}$ and $\alpha$ good and choose $A$ witnessing this; then we choose $\beta \in C$ a limit point of $A$ with cofinality $\rho$, so that $A \cap \beta$ witnesses $\beta$ is good.

Remark 2.8. It is easy to see that if $(\vec{\kappa}, \vec{f})$ and $(\vec{\kappa}, \vec{g})$ are two scales of length $\kappa^{+}$ for $\kappa$ in the same product $\prod_{i} \kappa_{i}$, then the set of good points for $\vec{f}$ is equal modulo clubs to the set of good points for $\vec{g}$.

Definition 2.9. A good scale for $\kappa$ is a scale for $\kappa$ in which, modulo the club filter, almost every point of cofinality greater than $\operatorname{cf}(\kappa)$ is good.

Now we look at a form of weak square (the approachability square $\mathrm{AP}_{\kappa}$ ) introduced by Shelah in [9]. $\mathrm{AP}_{\kappa}$ is substantially weaker than $\square_{\kappa}^{*}$.

Definition 2.10. $\mathrm{AP}_{\kappa}$ holds iff there exists $\left\langle C_{\alpha}: \alpha<\kappa^{+}\right\rangle$such that

1. $C_{\alpha}$ is a club subset of $\alpha$ of order type $\operatorname{cf}(\alpha)$ for each limit $\alpha$.

2. $C_{\gamma+1}$ is a subset of $\kappa^{+}$for each successor ordinal $\gamma+1$.

3. For a club of $\alpha<\kappa^{+}, \forall \beta<\alpha \exists \gamma<\alpha C_{\alpha} \cap \beta=C_{\gamma+1}$. 
We are interested in this principle mainly because of the following fact, which is Claim 4.4 in [4].

Fact 2.11. If $\mathrm{AP}_{\kappa}$ holds and $(\vec{\kappa}, \vec{f})$ is a scale then almost every point $\alpha$ with $\operatorname{cf}(\alpha)>$ $\operatorname{cf}(\kappa)$ is good for $\vec{f}$. That is, every scale is good.

We also collect here a few more facts about good points.

Fact 2.12. Let $\operatorname{cf}(\kappa)=\omega$ and $\kappa$ be strong limit with $2^{\kappa}>\kappa^{+}$. Then there exists a scale $(\vec{\kappa}, \vec{f})$ for $\kappa$ such that every point of uncountable cofinality is good for $\vec{f}$.

Fact 2.13. If $(\vec{\kappa}, \vec{f})$ is a scale for $\aleph_{\omega}$ and $\alpha<\aleph_{\omega+1}, \operatorname{cf}(\alpha)>2^{\aleph_{0}}$ then $\alpha$ is good for $\vec{f}$.

\section{The MAIN LEMMA}

In this section we will prove the key technical lemma of the paper, which connects the notion of "good point" with Questions 1 and 2 from the Introduction. We originally proved the lemma for points of cofinality $\lambda$, with the additional assumption that $\lambda$ is regular in $W$; Burke pointed out that it holds in the more general form given here.

Lemma 3.1. Let $V, W$ be inner models of set theory with $V \subseteq W$. Let $\kappa$ be singular in $V$, and $f i x(\vec{\kappa}, \vec{f})$ a scale of length $\kappa^{+}$for $\kappa$. Suppose that $\kappa_{V}^{+}=\lambda_{W}^{+}$ where $\lambda>\aleph_{0}$ and $W \models \operatorname{cf}(\lambda) \neq \operatorname{cf}(\kappa)$. Then there exists $\eta<\lambda$ such that for every $\delta \in R E G^{W} \cap(\eta, \lambda]$

$$
\left\{\gamma<\kappa_{V}^{+}: W \models " \operatorname{cf}(\gamma)=\delta \text { and } \gamma \text { is good for } \vec{f} "\right\}
$$

is non-stationary in $W$.

Proof. We work in $W$. Since $\kappa_{V}^{+}=\kappa_{W}^{+}$we will just denote this cardinal by " $\kappa^{+}$". Observe that $\mu={ }_{\text {def }} \operatorname{cf}_{W}(\kappa)<\lambda$ because $\lambda$ is the largest cardinal less than $\kappa^{+}$. $\operatorname{cf}_{W}\left(\operatorname{cf}_{V}(\kappa)\right)=\mu$, so we choose $C \subseteq \operatorname{cf}_{V}(\kappa)$ a cofinal set of order type $\mu$. $|\kappa|=\lambda$ so we may write $\kappa=\bigcup_{i<\lambda} X_{i}$ where $\vec{X}$ is an increasing sequence of sets with $\left|X_{i}\right|<\lambda$ for all $i<\lambda$.

Now for all $\alpha<\kappa^{+}, \operatorname{rge}\left(f_{\alpha}\lceil C) \subseteq \kappa=\bigcup_{i} X_{i}\right.$. Since $\mu \neq \operatorname{cf}(\lambda)$, for every $\alpha<\kappa^{+}$ there is $i<\lambda$ such that $\left\{n \in C: f_{\alpha}(n) \in X_{i}\right\}$ is unbounded in $\mu$. Hence we can fix $i<\lambda$ such that the set

$$
B={ }_{\text {def }}\left\{\alpha<\kappa^{+}:\left\{n \in C: f_{\alpha}(n) \in X_{i}\right\} \text { is unbounded in } \mu\right\}
$$

is unbounded in $\kappa^{+}$.

Now let $\eta=\max \left\{\left|X_{i}\right|, \mu\right\}$, and fix $\delta \in R E G^{W}$ with $\eta<\delta \leq \lambda$. Since $B$ is unbounded it has a club set of limit points; we claim that every limit point of $B$ with cofinality $\delta$ is not good for $\vec{f}$. To see this, suppose for a contradiction that $\gamma$ is a limit point of $B$ of cofinality $\delta$ and that $\gamma$ is good. This means that there is $A \subseteq \gamma$ unbounded and $m<\operatorname{cf}_{V}(\kappa)$ such that if $\alpha, \beta \in A, \alpha<\beta$ and $m<n<\operatorname{cf}_{V}(\kappa)$ then $f_{\alpha}(n)<f_{\beta}(n)$.

Now we build an increasing sequence of ordinals $\left\langle\gamma_{j}: j<\delta\right\rangle$ such that $\gamma_{2 j} \in A$ and $\gamma_{2 j+1} \in B$. Notice that $f_{\gamma_{2 j}}<^{*} f_{\gamma_{2 j+1}}<^{*} f_{\gamma_{2 j+2}}$ for each $j<\delta$. For each $j$ let us choose $n_{j}>m$ such that $n_{j} \in C$,

$$
f_{\gamma_{2 j}}\left(n_{j}\right)<f_{\gamma_{2 j+1}}\left(n_{j}\right)<f_{\gamma_{2 j+2}}\left(n_{j}\right),
$$

and $f_{\gamma_{2 j+1}}\left(n_{j}\right) \in X_{i}$. 
Since $\delta>\mu=|C|$ we can find $Z \subseteq \delta$ unbounded and a fixed $n \in C$ such that for all $j \in Z$

$$
f_{\gamma_{2 j}}(n)<f_{\gamma_{2 j+1}}(n)<f_{\gamma_{2 j+2}}(n)
$$

and $f_{\gamma_{2 j+1}}(n) \in X_{i}$. Now if $j, k$ are two elements of $Z$ with $j<k$ then

$$
f_{\gamma_{2 j+1}}(n)<f_{\gamma_{2 j+2}}(n) \leq f_{\gamma_{2 k}}(n)<f_{\gamma_{2 k+1}}(n) .
$$

This implies that $\left|\left\{f_{\gamma_{2 j+1}}(n): j \in Z\right\}\right|=|Z|=\delta$. However for each $j \in Z$ we have $f_{\gamma_{2 j+1}}(n) \in X_{i}$, and $\left|X_{i}\right|<\delta$, which is a contradiction. So almost every point of cofinality $\delta$ is not good and we have proved the claim of the lemma.

Remark 3.2. $R E G^{W} \cap(\eta, \lambda]$ is always non-empty. If $\lambda$ is regular in $W$ then $\lambda$ will do, and if $\lambda$ is singular then $\lambda$ is limit and we are guaranteed many suitable $\delta$.

\section{Exploiting the MAin LEMma}

Throughout this section our running assumptions are that $V \subseteq W, \kappa_{V}^{+}=\lambda_{W}^{+}$, $V \models " \kappa$ is a singular cardinal", and $W=\operatorname{cf}(\kappa) \neq \operatorname{cf}(\lambda)$.

Theorem 1. $W$ is not a $\kappa^{+}$-c.c. extension of $V$.

Proof. Fix $(\vec{\kappa}, \vec{f})$ a scale for $\kappa$. Applying Lemma 3.1, let $\delta \in R E G^{W}$ be such that in $W$ the set of good points with cofinality $\delta$ is non-stationary.

Let us define

$$
S=\left\{\gamma<\kappa^{+}: V \models " \operatorname{cf}(\gamma)=\delta \text { and } \gamma \text { is good for } \vec{f} "\right\} .
$$

By Lemma 2.7, $V \models$ "S is stationary". Since $\delta$ is regular in $W$ we know that

$$
V \models " \operatorname{cf}(\gamma)=\delta \text { and } \gamma \text { is good" } \Longrightarrow W \models " \operatorname{cf}(\gamma)=\delta \text { and } \gamma \text { is good", }
$$

and so by the choice of $\delta$ we have $W \models$ " $S$ is nonstationary". Since any $\kappa^{+}$c.c. forcing preserves stationary subsets of $\kappa^{+}, W$ is not a $\kappa^{+}$-c.c. generic extension of $V$.

We originally proved the following result under the additional assumptions that $\lambda$ is regular and $V \models \operatorname{cf}(\kappa)<\lambda$; Burke pointed out that it is true in the general form given here.

Theorem 2. $V \models$ "There is no good scale of length $\kappa^{+}$for $\kappa$ ".

Proof. Let $(\vec{\kappa}, \vec{f})$ be a good scale for $\kappa$ in $V$, and let $C$ be a club in $\kappa^{+}$such that $V \models$ "every point of $C$ with cofinality greater than $\operatorname{cf}(\kappa)$ is good".

Let $B$ be the unbounded set constructed in the proof of Lemma 3.1, and let $\delta \in$ $R E G^{W}$ be such that every limit point of $B$ with $W$-cofinality $\delta$ is not good and $\delta>\operatorname{cf}_{W}(\kappa)$.

Choose $\gamma \in C \cap \operatorname{acc}(B)$ such that $W=\operatorname{cf}(\gamma)=\delta$. By the choice of $B$ and $\delta, \gamma$ cannot be good. On the other hand $W=\operatorname{cf}(\gamma)=\delta>\operatorname{cf}(\kappa)$ so that $V \models \operatorname{cf}(\gamma) \neq$ $\operatorname{cf}(\kappa)$; if $V \models \operatorname{cf}(\gamma)<\operatorname{cf}(\kappa)$ then $\gamma$ is good by Remark 2.4 and if $V=\operatorname{cf}(\gamma)>\operatorname{cf}(\kappa)$ then $\gamma$ is good by the choice of $C$. Contradiction!

Theorem 3. Let $V \models \operatorname{cf}(\kappa)=\omega$. Then

1. $V=" \kappa$ is strong limit $\Longrightarrow 2^{\kappa}=\kappa^{+}$".

2. If $\lambda=\aleph_{1}^{W}$ then ${ }^{\omega} \kappa \cap W \supsetneq{ }^{\omega} \kappa \cap V$. 
Proof. The first claim follows immediately from Fact 2.12 and Theorem 2 . For the second claim, suppose for a contradiction that ${ }^{\omega} \kappa \cap W={ }^{\omega} \kappa \cap V$ and fix (in $V$ ) a scale $(\vec{\kappa}, \vec{f})$ of length $\kappa^{+}$for $\kappa$.

Now $W \models \operatorname{cf}\left(\kappa_{n}\right)>\omega$ for all $n<\omega$ and $W \models " \vec{f}$ is cofinal in $\prod_{n} \kappa_{n}$ ". This is enough for us to imitate the proof of Lemma 2.7 and to prove (by building in $W$ an $\aleph_{1}$-chain of countable structures) that

$$
W \models "\left\{\gamma<\kappa^{+}: \operatorname{cf}(\gamma)=\aleph_{1} \text { and } \gamma \text { is } \operatorname{good}\right\} \text { is stationary". }
$$

This contradicts Lemma 3.1.

Theorem 4. If $\kappa=\aleph_{\omega}^{V}$ then $\lambda \leq\left(2^{\aleph_{0}}\right)_{V}$.

Proof. Fix $(\vec{\kappa}, \vec{f})$ a scale of length $\kappa^{+}$for $\kappa$. Recall that by Fact 2.13 all points of cofinality greater than $2^{\aleph_{0}}$ are good for $\vec{f}$.

Suppose for a contradiction that $\lambda>\left(2^{\aleph_{0}}\right)_{V}$. Using Lemma 3.1 find $\gamma$ such that $W \models \operatorname{cf}(\gamma)>\left(2^{\aleph_{0}}\right)_{V}$, and $\gamma$ is not good for $\vec{f}$. Now $V \models \operatorname{cf}(\gamma)>\left(2^{\aleph_{0}}\right)_{V}$, contradicting 2.13 .

\section{Concluding Remarks}

The problems discussed in this paper are connected with some other open problems in set theory.

5.1. Chang's conjecture. The consistency of " $\left(\aleph_{\omega+1}, \aleph_{\omega}\right) \rightarrow\left(\aleph_{2}, \aleph_{1}\right)$ " is open. If this holds then $S={ }_{\operatorname{def}}\left\{X \subseteq \aleph_{\omega+1}: \operatorname{ot}(X)=\aleph_{2}\right\}$ is stationary, and supposing that there exists a Woodin cardinal $\delta$ we can force with Woodin's stationary tower forcing [12] below the condition $S$. It is easy to see that $S \Vdash \aleph_{\omega+1}^{V}=\aleph_{2}^{V[G]}$.

5.2. Good points. Let $(\vec{\kappa}, \vec{f})$ be a scale of length $\aleph_{\omega+1}$ for $\aleph_{\omega}$. It is open whether the set of points $\left\{\alpha<\aleph_{\omega+1}: \operatorname{cf}(\alpha)>\aleph_{1}\right.$ and $\alpha$ is not good $\}$ can ever be stationary. If this set cannot be stationary then Lemma 3.1 implies that $\aleph_{\omega+1}$ can only be collapsed to $\aleph_{1}$ or $\aleph_{2}$.

This problem is also connected with " $\left(\aleph_{\omega+1}, \aleph_{\omega}\right) \rightarrow\left(\aleph_{2}, \aleph_{1}\right)$ ", because if this holds then by Claim 4.3 in [4] there is a stationary set of cofinality $\aleph_{2}$ points which are not good. It is known [4] that if MM holds or $\left(\aleph_{\omega+1}, \aleph_{\omega}\right) \rightarrow\left(\aleph_{1}, \aleph_{0}\right)$ then the set $\left\{\alpha<\aleph_{\omega+1}: \operatorname{cf}(\alpha)=\aleph_{1}\right.$ and $\alpha$ is not good $\}$ is stationary. The statement "( $\left.\aleph_{\omega+1}, \aleph_{\omega}\right) \rightarrow\left(\aleph_{1}, \aleph_{0}\right) "$ is known [5] to be consistent.

5.3. Saturated ideals. Burke and Matsubara have pointed out that the results in this paper can be used to show that the nonstationary ideal on $\mathcal{P}_{\kappa} \lambda$ is not saturated for certain values of $\kappa$ and $\lambda$.

\section{REFERENCES}

[1] L. Bukovský and E. Copláková-Hartová, Minimal collapsing extensions of models of ZFC. Annals of Pure and Applied Logic, 46:265-298, 1990. MR 92e:03077

[2] M. Burke and M. Magidor, Shelah's pcf theory and its applications. Annals of Pure and Applied Logic, 50:207-254, 1990. MR 92f:03053

[3] J. Cummings, M. Foreman and M. Magidor. Scales, squares and stationary reflection. In preparation.

[4] M. Foreman and M. Magidor, A very weak square principle. To appear.

[5] J. P. Levinski, M. Magidor and S. Shelah, Chang's conjecture for $\aleph_{\omega}$. Israel Journal of Mathematics, 69:161-172, 1990. MR 91g:03071 
[6] W. J. Mitchell and E. Schimmerling, Covering without countable closure. Mathematical Research Letters, 2:595-609, 1995. MR 96k:03123

[7] W. J. Mitchell, E. Schimmerling,and J. R. Steel, The Covering Lemma up to a Woodin cardinal. To appear in Annals of Pure and Applied Logic.

[8] E. Schimmerling, Combinatorial principles in the core model for one Woodin cardinal. Annals of Pure and Applied Logic, 74:153-201, 1995. MR 96f:03041

[9] S. Shelah, On successors of singulars. In Logic Colloquium '78, pp 357-380, Amsterdam, 1979, North-Holland. MR 82d:03079

[10] S. Shelah, Proper Forcing. Berlin, 1982, Springer-Verlag. MR 84h:03002

[11] S. Shelah, Cardinal Arithmetic. Oxford, 1994, Oxford University Press. MR 96e:03001

[12] H. Woodin, Supercompact cardinals, sets of reals and weakly homogeneous trees. Proceedings of the National Academy of Sciences of the USA, 85:6587-6591. MR 89m:03040

Department of Mathematics 2-390, Massachusetts Institute of Technology, CamBRIDGe, MASSACHUSETTS 02139

E-mail address: cummings@math.mit.edu

Current address: Department of Mathematical Sciences, Carnegie Mellon University, Pittsburgh, Pennsylvania 15213-3890

E-mail address: jcumming@andrew.cmu.edu 